\title{
Influencia del sistema de salud de procedencia y otros factores en el resultado del reemplazo valvular mitral
}

\author{
SERGIO MORÁN, CECILIA MUÑOZ, BERNARDITA GARAYAR, \\ RICARDO ZALAQUETT, MANUEL J. IRARRÁZAVAL, \\ PEDRO BECKER, RODRIGO GONZÁLEZ
}

\section{Influence of the referring health care systems and other factors on the results of mitral valve replacement}

Background: There is no consensus regarding which risk factors influence the outcome of mitral valve replacement. Aim: To study the effects of the referring health care system and other factors on the results of mitral replacement. Patients and Methods: We included 632 patients operated between 1990 and 2010 receiving the St Jude prosthesis. Patients were divided into three groups, group 1 composed by 180 patients coming from the Public System, group 2 composed by 182 patients coming from the University System and group 3 composed by 270 patients coming from the Private System. Results: Overall operative mortality was 4.3\%. There was no difference between groups in mortality. Factors responsible for operative mortality were: emergency operation (Odds Ratio $(O R):$ 5.6. $P<0.01)$ and left ventricular function (according to ejection fraction) grade III to IV (OR: 2.5. $p=0.048)$. Actuarial survival rates at $1,5,10,15$ and 20 years were $95 \%, 87 \%, 76 \%, 61 \%$ and $41 \%$, respectively. Risk factors for long-term mortality were diabetes (OR: 3.3. $p<0.01$ ), left ventricular function grades III-IV (OR: 2.6p $<0.01)$, New York Heart Association functional class III to IV (OR: 2, 1. $p<0.005)$ and male sex (OR: 1, 5. $p<0.032)$. Conclusions: Referring health care system and type of surgery do not constitute a risk factor for mitral replacement. Risk factors were: emergency surgery, ventricular function grades III-IV, diabetes, functional capacity class III-IV and male sex. Integration of public and private health care systems in a university hospital setting achieves excellent outcomes for complex pathology.

(Rev Med Chile 2013; 141: 861-869).

Key words: Delivery of health care; Heart valve prosthesis implantation; Mitral valve; Risk factors.

4 1 reemplazo valvular mitral ha significado una mejoría muy importante en la calidad de vida y en la supervivencia de pacientes con enfermedad valvular grave. Si bien no existe unanimidad sobre los factores de riesgo más relevantes con respecto a los resultados de la cirugía, se ha planteado que los más importantes dependen de características clínicas del paciente, tipo de

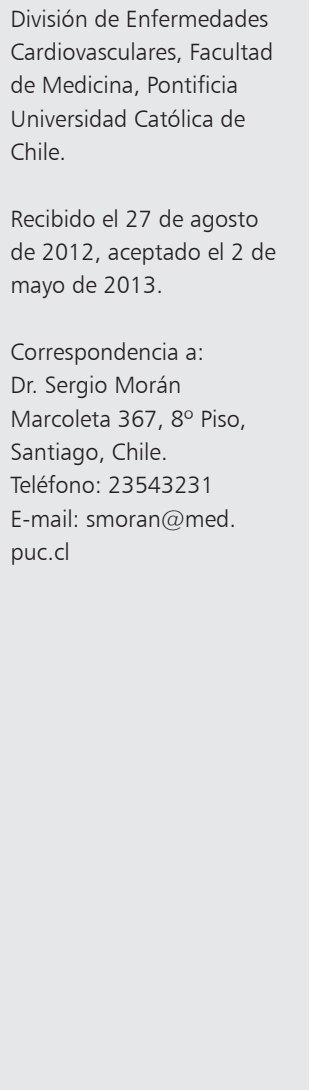

prótesis, operación efectuada y sistema de salud al que el paciente consulta ${ }^{1-3}$.

Con el objeto de estudiar cuales factores de riesgo influyen en los resultados del reemplazo de la válvula mitral, decidimos analizar nuestra experiencia con pacientes intervenidos entre 1990 y 2010. Dado que ellos provenían del sistema público financiados por el Fondo Nacional de Salud 
(FONASA) y del sistema privado financiados por Instituciones de Salud Previsional (Isapres), nos propusimos evaluar, desde nuestra perspectiva de hospital universitario, un factor importante pero poco estudiado, el sistema de salud al que consulta el paciente.

Como actualmente existe discusión sobre carencias y listas de espera en los servicios públicos ${ }^{4-6}$ es posible que un análisis de nuestros resultados logre aportar argumentos que favorezcan colaboraciones entre los sistemas de salud para otorgar un servicio de calidad a todos los pacientes ${ }^{7,8}$.

\section{Pacientes y Métodos}

Durante el período comprendido entre el $1^{\circ} \mathrm{de}$ enero de 1990 y el 31 de diciembre de 2010, mil treinta pacientes recibieron un reemplazo de la válvula mitral en nuestra institución. Se incluyeron en el estudio los mayores de 15 años que recibieron la prótesis de St. Jude ${ }^{\circledR}$ para el reemplazo valvular (St. Jude Medical Inc., St. Paul, Minn. EE.UU.); aquellos en los que además se realizaron operaciones combinadas de reemplazo o reparación de otra válvula(s); los que recibieron concomitantemente puentes aortocoronarios, y por último, los intervenidos electivamente y de urgencia. Se excluyeron los pacientes que recibieron otro tipo de prótesis para tener una población homogénea y los extranjeros, por no tener información de su evolución alejada.

Los pacientes fueron intervenidos por un mismo equipo y con igual técnica. La operación se realizó bajo circulación extracorpórea con oxigenador de membrana e hipotermia sistémica a $28-30^{\circ} \mathrm{C}$. La protección miocárdica se efectuó con solución cardiopléjica cristaloide infundida a $4^{\circ} \mathrm{C}$ en forma anterógrada y retrograda ${ }^{9}$. Los datos de la operación y características demográficas del paciente fueron colectados prospectivamente en la base de datos del Servicio de Cirugía Cardiovascular del Hospital Clínico de la Pontificia Universidad Católica de Chile. Además se revisaron las fichas clínicas y protocolos operatorios de todos los pacientes y se analizaron factores clínicos, de laboratorio y co-morbilidades según criterios internacionales ${ }^{10,11}$. La severidad de los síntomas fue clasificada según la New York Heart Association $^{12}$. La función ventricular izquierda evaluada mediante ecocardiograma fue clasificada de acuerdo al American College of Cardiology ${ }^{13}$ : grado I función normal, fracción de eyección $(\mathrm{FE})>50$; grado II leve disminución de la función ventricular FE > 41-50; grado III moderada disminución FE $>30-40$ y grado IV severa disminución $\mathrm{FE}<30$. La supervivencia alejada se objetivó accediendo al Registro Civil e Identificación de Chile y a los certificados de defunción cuando correspondió. Todos los pacientes recibieron tratamiento anticoagulante con Acenocumarol ${ }^{\circledR}$ ajustando la dosis para obtener un INR (International Normalized Ratio) entre 2,5 y 3,5 .

Para evaluar los resultados según sistemas de salud de procedencia, los pacientes se dividieron en tres grupos. Grupo 1 Sistema Público: pacientes derivados desde el Hospital Sótero del Río gracias a un convenio docente-asistencial existente entre el Ministerio de Salud y la Pontificia Universidad Católica de Chile, y de otros hospitales públicos mediante el sistema de gestión de camas. Grupo 2 Sistema Universitario: pacientes que consultaron en el policlínico de la Red de Salud UC en el Centro de Diagnóstico San Joaquín y que pertenecían tanto al sistema público como privado mediante convenios específicos. Grupo 3 Sistema Privado: pacientes referidos de las consultas privadas de cardiólogos de nuestro hospital y otros cardiólogos del país, que se financiaron con el sistema de seguros Isapres.

\section{Estadística}

Los resultados están expresados como promedio \pm desviación estándar. Las variables categóricas fueron comparadas usando tablas de contingencia o test exacto de Fisher. Las variables continuas se expresan en promedio y desviación estándar y son comparadas usando t de Student. Se realizó análisis uni y multivariado con regresión logística para identificar los factores asociados a mortalidad inmediata y alejada. Las curvas de supervivencia global y libre de eventos se construyeron usando el método de Kaplan-Meier. Los grupos fueron comparados usando el test de Log-Rank.

Los factores predictores independientes fueron determinados por medio de análisis multivariado de Cox. Un valor $\mathrm{p}<0,05$ fue considerado significativo. Se utilizó programa estadístico SPSS 17.0.

\section{Resultados}

Durante el período del estudio, 632 pacientes sometidos a reemplazo valvular mitral cumplieron con los criterios de inclusión: 180 pacientes $(28,5 \%)$ pertenecieron al Grupo 1 Sistema Público; 
Procedencia del paciente en resultado de reemplazo mitral - S. Morán et al

$182(28,8 \%)$ al Grupo 2 Sistema Universitario y 270 (42,7\%) al Grupo 3 Sistema Privado.

Los datos demográficos y los hallazgos clínicos están resumidos en la Tabla 1 . El grupo 1 fue más joven $(\mathrm{p}<0,005)$, tuvo menos cirugías previas, sin embargo, tuvo más fibrilación auricular y más hipertensión que los otros dos grupos ( $<<0,001)$. El grupo 3 en cambio fue de mayor edad, tuvo más enfermedad coronaria y ritmo sinusal $(p<0,001)$.

La etiología de la lesión valvular fue reumática en 535 (84,6\%); degenerativa en $41(6,4 \%)$; isquémica en 24 (3,8\%); infecciosa en $14(2,2 \%)$ y misceláneas en $18(2,8 \%)$ (miocardiopatía hipertrófica/dilatada 8 , congénita 2 , traumática 2, no identificada 6). Según el tipo de operación efectuada, 264 recibieron un reemplazo valvular mitral único (RVM), 141 un RVM más plastia tricúspide y 227 un RVM más otra operación: reemplazo valvular aórtico (RVA) en 171, más plastia tricúspide 48 , puentes aortocoronarios
(PAC) en 44 pacientes y reemplazo tricúspide en 13. Treinta y tres pacientes fueron operados de urgencia $(5,2 \%)$. Treinta y uno por insuficiencia mitral aguda (isquémica 12, disfunción de prótesis 9 , endocarditis infecciosa 7 y degenerativa 3 ) y 2 por embolias a repetición. No hubo diferencias entre los grupos según la etiología, tipo de operación ni carácter de urgencia.

La mortalidad operatoria global hasta 30 días o hasta el término de la hospitalización del paciente si esta fue mayor, fue $4,3 \%$. Veintisiete pacientes fallecieron; 18 por causas cardiacas y 9 por causas no cardiacas. Las causas de mortalidad de cada grupo están resumidas en la Tabla 2. La mortalidad operatoria fue de 3,4\% para 264 pacientes con RVM único; 4,9\% para 141 pacientes con RVM más plastia tricúspide y $4,8 \%$ para 227 pacientes con RVM más otra operación. No hubo diferencias estadísticamente significativas entre los grupos, divididos tanto por sistema de atención,

Tabla 1. Características clínicas y demográficas de los pacientes agrupados según sistema de salud que consultaron

\begin{tabular}{|c|c|c|c|c|c|}
\hline Características & $\begin{array}{l}\text { Grupo } 1 \\
\text { Sistema } \\
\text { Público }\end{array}$ & $\begin{array}{c}\text { Grupo } 2 \\
\text { Sistema } \\
\text { Universitario }\end{array}$ & $\begin{array}{l}\text { Grupo } 3 \\
\text { Sistema } \\
\text { Privado }\end{array}$ & Total & $\mathbf{p}$ \\
\hline Pacientes \% & $180(28,5)$ & $182(28,8)$ & $270(42,7)$ & $632(100)$ & \\
\hline $\begin{array}{l}\text { Edad años } \pm \text { DS } \\
\text { (margen) }\end{array}$ & $\begin{array}{l}52,7 \pm 11,6 \\
(16-74)\end{array}$ & $\begin{array}{c}55,7 \pm 11,9 \\
(17-81)\end{array}$ & $\begin{array}{c}56,7 \pm 11,7^{*} \\
(18-83)\end{array}$ & $\begin{array}{c}55,3 \pm 11,9 \\
(16-83)\end{array}$ & 0,005 \\
\hline $\begin{array}{l}\text { Mujeres (\%) } \\
\text { Hombres (\%) }\end{array}$ & $\begin{array}{r}123(68,3) \\
57(31,7)\end{array}$ & $\begin{array}{r}130(71,4) \\
52(28,6)\end{array}$ & $\begin{array}{l}169(62,6) \\
101(37,4)\end{array}$ & $\begin{array}{l}422(66,8) \\
210(33,2)\end{array}$ & ns \\
\hline Diabetes mellitus (\%) & $22(12,2)$ & $23(12,6)$ & $24(8,9)$ & $69(10,9)$ & ns \\
\hline Hipertensión arterial (\%) & $67(37,2)^{*}$ & $45(24,7)$ & $48(17,8)$ & $160(25,3)$ & 0,001 \\
\hline Tabaquismo (\%) & $49(27,2)$ & $40(22)$ & $85(31,5)$ & $174(27,5)$ & ns \\
\hline Insuficiencia renal (\%) & $16(8,9)$ & $23(12,6)$ & $32(11,9)$ & $71(11,2)$ & ns \\
\hline Enfermedad coronaria (\%) & $16(8,9)$ & $14(7,7)$ & $42(15,6)^{*}$ & $72(11,4)$ & 0,019 \\
\hline $\begin{array}{l}\text { CF NYHA I - II (\%) } \\
\text { CF NYHA III - IV (\%) }\end{array}$ & $\begin{array}{r}26(14,4) \\
154(85,5)\end{array}$ & $\begin{array}{r}43(23,6) \\
139(76,3)\end{array}$ & $\begin{array}{r}66(24,4) \\
204(75,5)\end{array}$ & $\begin{array}{l}135(21,3) \\
497(78,6)\end{array}$ & 0,036 \\
\hline $\begin{array}{l}\text { Función VI Grado I - II (\%) } \\
\text { Función VI Grado III - IV (\%) }\end{array}$ & $\begin{array}{r}154(85,5) \\
26(14,4)\end{array}$ & $\begin{array}{r}164(90,1) \\
18(9,9)\end{array}$ & $\begin{array}{r}236(87,4) \\
34(12,5)\end{array}$ & $\begin{array}{r}557(88,1) \\
75(11,9)\end{array}$ & ns \\
\hline $\begin{array}{l}\text { Fibrilación auricular (\%) } \\
\text { Fibrilación auricular paroxística (\%) } \\
\text { Ritmo sinusal (\%) }\end{array}$ & $\begin{array}{l}129(71,7)^{*} \\
11(6,1) \\
36(20)\end{array}$ & $\begin{array}{r}113(62,1) \\
15(8,2) \\
50(27,5)\end{array}$ & $\begin{array}{c}140(51,9) \\
15(5,6) \\
110(40,7)^{*}\end{array}$ & $\begin{array}{r}382(60,4) \\
41(6,5) \\
196(31)\end{array}$ & 0,001 \\
\hline Plastía mitral percutánea (\%) & $18(10)$ & $17(9,3)$ & $34(12,6)$ & $69(10,9)$ & ns \\
\hline Plastía mitral quirúrgica (\%) & $31(17,2)$ & $40(22)$ & $54(20)$ & $125(19,8)$ & ns \\
\hline Cirugía cardiaca previa (\%)** & $41(22,8)^{*}$ & $78(42,9)$ & $99(36,7)$ & $218(34,5)$ & $<0,001$ \\
\hline
\end{tabular}

*Estadísticamente significativo $(p<0,05)$. **Incluye plastia mitral quirúrgica, RVM, RVA o PAC previo. 
como por tipo de operación. Tabla 3. En cambio, los 33 pacientes operados de urgencia tuvieron una mortalidad significativamente mayor, 7 casos $(21,2 \%)$, que los 599 intervenidos electivamente, 20 casos $(3,3 \%)(\mathrm{p}=0,001)$.

Los factores que influyeron significativamente en la mortalidad operatoria según análisis uni y multivariado están descritos en la Tabla 4. De los cinco factores obtenidos por análisis univariado, sólo la cirugía de urgencia y la función ventricular grados III y IV tuvieron significancia en el análisis multivariado.

En cuanto a los resultados alejados incluyendo la mortalidad operatoria, la supervivencia fue determinada mediante certificados del Registro Civil e Identificación. El promedio de tiempo entre la cirugía y la constatación de sobrevida o fallecimiento del paciente fue de 8,9 años. La sobrevida actuarial a 1, 5, 10, 15 y 20 años fue de $95 \%, 87 \%$, $76 \%, 61 \%$ y $41 \%$ respectivamente. No se encontra-

Tabla 2. Causas de mortalidad operatoria

\begin{tabular}{|lccc|}
\hline & $\begin{array}{c}\text { Grupo 1 } \\
\text { Sistema público } \\
(\mathbf{n}=\mathbf{1 8 0})\end{array}$ & $\begin{array}{c}\text { Grupo 2 } \\
\text { Sistema universitario } \\
(\mathbf{n = 1 8 2})\end{array}$ & $\begin{array}{c}\text { Grupo } \mathbf{3} \\
\text { Sistema privado } \\
\text { (n = 270) }\end{array}$ \\
\hline Cardiaca (débito bajo) & 3 & 4 & 11 \\
No Cardiaca & 1 & 5 & 3 \\
\hline Sepsis & 1 & 2 & 2 \\
AVC & - & 3 & - \\
\hline Insuficiencia respiratoria & - & - & 1 \\
\hline
\end{tabular}

AVC = accidente vascular cerebral.

Tabla 3. Mortalidad operatoria según sistema de salud que el paciente consulta y según tipo de operación efectuada $(p=N S)$

\begin{tabular}{|lcccc|}
\hline Tipo de operación & $\begin{array}{c}\text { Grupo 1 } \\
\text { Sistema público } \\
(\mathbf{n}=\mathbf{1 8 0})\end{array}$ & $\begin{array}{c}\text { Grupo 2 } \\
\text { Sistema universitario } \\
(\mathbf{n}=\mathbf{1 8 2})\end{array}$ & $\begin{array}{c}\text { Grupo 3 } \\
\text { Sistema privado } \\
(\mathbf{n}=\mathbf{2 7 0})\end{array}$ & $\begin{array}{c}\text { Mortalidad } \\
\text { operatoria } \\
\text { (n = 27) }\end{array}$ \\
$\begin{array}{l}\text { RVM único } \\
(\mathrm{N}=264)\end{array}$ & 53 & 88 & 123 & $9(3,4 \%)$ \\
$\begin{array}{l}\text { RVM + plastía tricúspide } \\
(\mathrm{N}=141)\end{array}$ & 52 & 41 & 48 & $7(4,9 \%)$ \\
$\begin{array}{l}\text { RVM + otra op. } \\
(\mathrm{N}=227)\end{array}$ & 75 & 53 & 99 & $11(4,8 \%)$ \\
$\begin{array}{l}\text { Mortalidad operatoria } \\
(\mathrm{N}=27)\end{array}$ & $4(2,2 \%)$ & $9(4,9 \%)$ & $14(5,2 \%)$ & \\
\hline
\end{tabular}

Tabla 4. Análisis uni y multivariado para factores de riesgo de mortalidad operatoria

\begin{tabular}{|lccc|}
\hline Factor & P univariado & P multivariado & Odds Ratio (CI) \\
\hline Cirugía de urgencia & $<0,001$ & $<0,001$ & $5,6(2,146-14,829)$ \\
\hline Disfunción VI grado III - IV & 0,013 & 0,048 & $2,5(1,008-6,065)$ \\
\hline Cirugía cardiaca previa & $<0,001$ & NS & NS \\
\hline Capacidad Funcional III - IV & $<0,001$ & NS & NS \\
\hline Fibrilación auricular & $<0,001$ & NS & NS \\
\hline
\end{tabular}


ron diferencias significativas entre los tres grupos de sistema de atención (Figura 1). Las causas de mortalidad alejada fueron cardiacas $(42,4 \%)$, no cardiacas $(38,2 \%)$ y secundarias a complicaciones derivadas de la prótesis $(19,4 \%)$ : necesidad de re operación por disfunción protésica secundaria a desprendimiento peri-prostético o endocarditis infecciosa y complicaciones del tratamiento anticoagulante. Entre las causas cardiacas destaca la insuficiencia cardiaca por progresión del daño ventricular. Tabla 5. La supervivencia alejada según tipo de operación efectuada tampoco mostró diferencias significativas (Figura 2).

Los factores de riesgo relacionados a una menor sobrevida alejada en el análisis univariado y multivariado están descritos en la Tabla 6. De los factores ajustados, la diabetes, función ventricular grados III y IV, capacidad funcional III y IV según la New York Heart Association (NYHA) y sexo masculino tuvieron diferencia significativa.
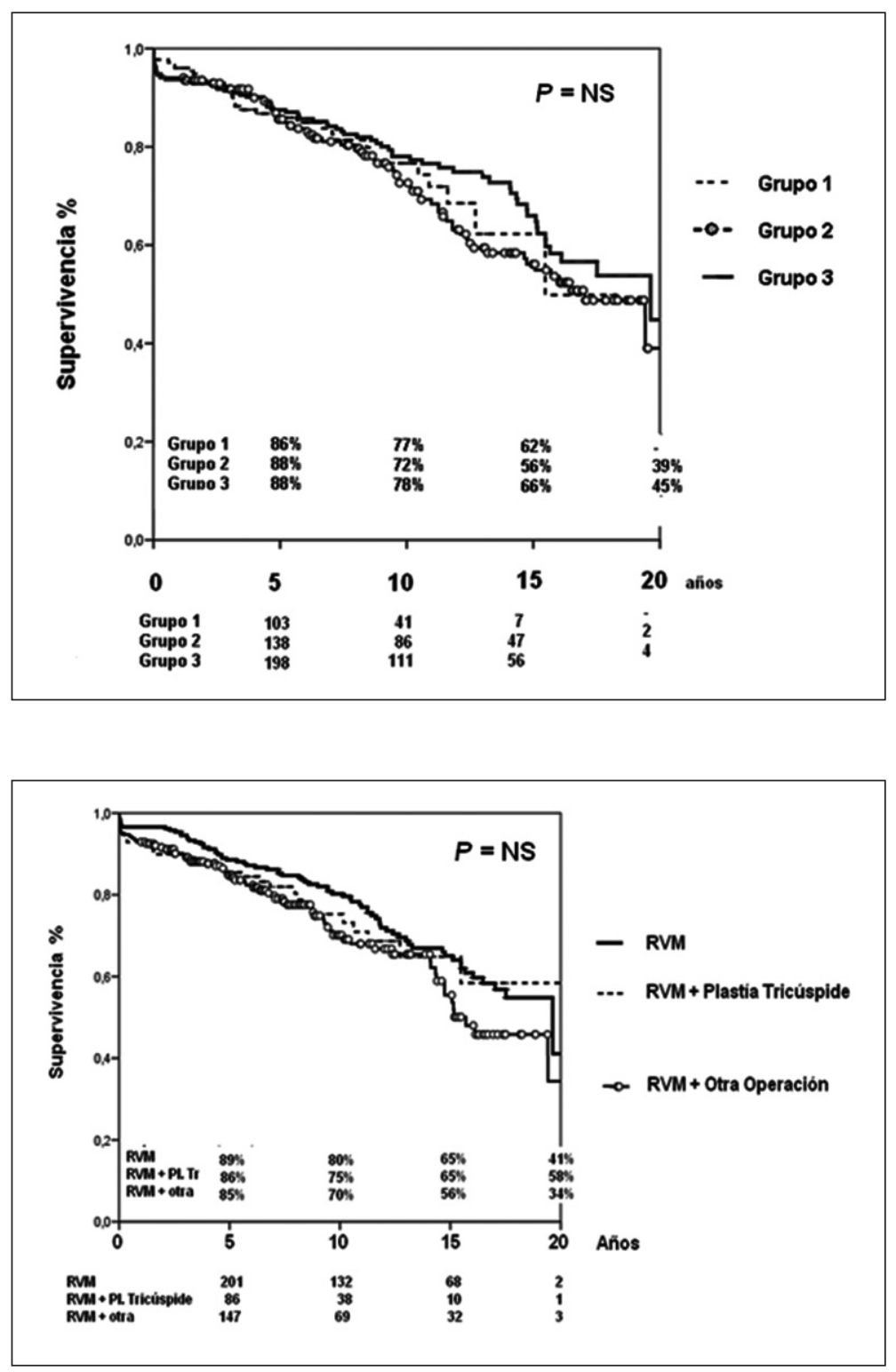

Figura 1. Supervivencia según sistema de Salud.
Figura 2. Supervivencia según operación realizada. 
Procedencia del paciente en resultado de reemplazo mitral - S. Morán et al

Tabla 5. Causas de mortalidad alejada

\begin{tabular}{|c|c|c|c|c|c|c|}
\hline Cardiacas & $\mathbf{n}$ & No cardiacas & $\mathbf{n}$ & Protésicas & & $\mathbf{n}$ \\
\hline Insuficiencia cardiaca & 45 & Insuficiencia respiratoria & 18 & Hemorragia & & 17 \\
\hline Infarto miocárdico & 11 & Cáncer & 12 & Cerebral & $\begin{array}{r}12 \\
2\end{array}$ & \\
\hline \multirow[t]{7}{*}{ Fibrilación ventricular/paro cardiaco } & 5 & Sepsis & 11 & Retroperitoneal & 1 & \\
\hline & & & & Hematoma sub dural & 2 & \\
\hline & & Falla orgánica múltiple & 6 & Disfunción protésica & & 6 \\
\hline & & Suicidio & 3 & Embolia cerebral & & 5 \\
\hline & & Insuficiencia hepática & 3 & & & \\
\hline & & Shock hipoglicémico & 1 & & & \\
\hline & & Trombosis mesentérica & 1 & & & \\
\hline Total & 61 & Total & 55 & Total & & 28 \\
\hline
\end{tabular}

Tabla 6. Análisis uni y multivariado de factores de riesgo para mortalidad alejada

\begin{tabular}{|lccc|}
\hline Factor & p univariado & p multivariado & Odds ratio $(\mathbf{C I})$ \\
\hline Diabetes mellitus & $<0,001$ & $<0,001$ & $3,3(2,153-5,305)$ \\
\hline Función VI grados III-IV & $<0,001$ & $<0,001$ & $2,6(1,687-3,974)$ \\
\hline Capacidad funcional III-IV & $<0,001$ & 0,005 & $2,1(1,245-3,414)$ \\
Sexo masculino & 0,002 & 0,032 & $1,5(1,035-2,184)$ \\
Insuficiencia renal & 0,014 & NS & NS \\
\hline
\end{tabular}

\section{Discusión}

Nuestros principales resultados muestran que los factores de riesgo significativos para mortalidad operatoria fueron: operación de urgencia y función ventricular izquierda grados III y IV. Para la mortalidad alejada, éstos fueron: diabetes mellitus, función ventricular grados III y IV, capacidad funcional clases III y IV y sexo masculino, concordando con otros autores ${ }^{14-18}$. Estos hallazgos permitirían simplificar la colección de datos para evaluar la mortalidad operatoria y alejada y hacer comparaciones válidas. Otros factores considerados de riesgo anteriormente, como el tipo de operación o el tipo de prótesis, han perdido valor pronóstico gracias al progreso de la técnica quirúrgica, la calidad de las prótesis, la protección miocárdica y la experiencia de los equipos quirúrgicos ${ }^{2,10,16,17}$.

El sistema de salud de procedencia no determinó diferencias en la mortalidad operatoria ni la supervivencia alejada entre los tres grupos analiza- dos, a diferencia de lo propuesto por Rahimtoola y Grunkemeier et al, quienes sostienen que el sistema de salud donde el paciente consulta es un factor de riesgo para la cirugía cardiaca ${ }^{1-3}$. Esto es relevante para nuestro país con un sistema de salud mixto. En Chile podemos distinguir los siguientes sistemas de prestaciones de salud: el sistema nacional de servicios de salud, las clínicas privadas, el sistema universitario con sus dos hospitales clínicos y consultorios, el de las FFAA y sus hospitales y por último, las mutuales de seguridad y sus hospitales ${ }^{19}$. Sabemos que los sistemas privados seleccionan por capacidad de pago y que los sistemas públicos presentan falencias de especialistas e infraestructura para atender la demanda de patologías complejas ${ }^{4-8,20}$. Esto permite plantear que al utilizar en forma complementaria los recursos de salud existentes en el país, se podrían beneficiar todos los que lo necesitan, independientemente de su condición socio económica, ya que existe capacidad no utilizada en el sector universitario y privado nacional. La colaboración entre los dis- 
tintos sistemas de prestaciones de salud puede así contribuir a reducir la inequidad en salud, meta prioritaria del gobierno de Chile, que ha mostrado avances, pero necesita progresar con mayor celeridad, al igual que en otros países ${ }^{7,8,20,21}$.

Una vez que el paciente del sistema público fue referido a nuestro servicio para su intervención, este fue operado prontamente sin diferencia con los pacientes del sistema universitario y de seguros privados, aprovechando nuestras ventajas organizacionales y de infraestructura. Una colaboración más extendida podría evitar largos tiempos de espera en el sistema público, como también se han visto en países desarrollados ${ }^{20,22}$. La mayor prevalencia de hipertensión arterial en el grupo 1, a pesar de su menor edad, coincide con lo detectado por la Encuesta Nacional de Salud 2009-2010 23, lo que apoya la representatividad de nuestra clasificación. También este grupo presenta una mayor incidencia de fibrilación auricular, lo que podría explicarse por deterioro cardiaco debido a los tiempos de espera para su atención, comparado con los otros grupos 2 y 3 que no tuvieron tiempo de espera desde la primera consulta hasta la operación misma.

Otro hallazgo relevante del presente estudio muestra una disminución de la mortalidad operatoria con respecto a comunicaciones previas ${ }^{17,24-27}$. Actualmente, nuestros resultados para pacientes sometidos a RVM sólo o asociado a plastia tricúspide y a RVM más otra operación, son similares a lo reportados por la Sociedad de Cirugía Torácica de Norteamérica y otros autores en pacientes de igual riesgo ${ }^{28-31}$. Así, la mortalidad operatoria para RVM fue 3,4\%; para RVM más plastia tricúspide fue $4,9 \%$ y para RVM más otra operación fue $4,8 \%$, sin diferencias significativas entre ellas. Esto constituye una mejoría con respecto a lo comunicado anteriormente, sobre todo considerando que 270 pacientes $(42,5 \%)$ recibieron una intervención quirúrgica más compleja, tradicionalmente asociada a mortalidades mayores que el reemplazo univalvular. Además 7\% de nuestros pacientes requirió PAC y 5,2\% de ellos fueron operados de urgencia, lo que constituye un mayor riesgo ${ }^{18,28}$.

$\mathrm{Al}$ dividir por sistema de salud, la mortalidad operatoria fue 2,2\% para el grupo 1; 4,9\% para el grupo 2 y $5,2 \%$ para el grupo 3 , sin diferencias significativas entre ellos, no obstante que las edades promedio de cada grupo fueron significativamente diferentes: $52,7 \pm 11,6 ; 55,7 \pm 11,9$ y $56,7 \pm 11,7$ años respectivamente $(\mathrm{p}<0,005)$. Además el grupo 3 presentó más enfermedad coronaria $(\mathrm{p}<0,01)$. Estas diferencias podrían causar mayor mortalidad. Sin embargo, esto no se observó, lo que traduciría un progreso importante y permitiría indicar la operación a pacientes más añosos y con co-morbilidades más graves, obteniendo buenos resultados ${ }^{29}$. Jamieson et al. utilizando la base de datos de la Sociedad de Cirugía Torácica con 86.500 pacientes de diversas instituciones acreditadas de Norteamérica durante 10 años, demostraron que se puede predeterminar la mortalidad operatoria según factores de riesgo operatorio de pacientes candidatos a cirugía cardiaca $^{28}$. Con esta evidencia se acepta actualmente que la relación entre la mortalidad observada y la deducida de estos parámetros es un muy buen índice de calidad del servicio médico prestado, que además permite comparar resultados con otros grupos quirúrgicos ${ }^{10,28,32}$.

Otra consideración importante que se desprende de este estudio es que, siendo la severidad de los síntomas y el deterioro de la función ventricular factores que elevan la mortalidad operatoria y disminuyen la supervivencia alejada, debiéramos indicar el reemplazo valvular más precozmente, como lo establecen las actuales guías de la ACC/ $\mathrm{AHA}^{33}$ y lo reportan autores recientes ${ }^{15,34}$.

En cuanto a la prótesis usada, estudios multicéntricos y prospectivos no han mostrado diferencias significativas entre los diferentes tipos de prótesis con respecto a mejoría sintomática, incidencia de complicaciones y supervivencia alejada ${ }^{2,27,35,36}$. Nosotros decidimos utilizar una prótesis mecánica en vez de biológica, dada la edad y la alta incidencia de fibrilación auricular de nuestra serie. La prótesis más usada para el reemplazo valvular mitral hoy es la St. Jude ${ }^{\circledR}$, prótesis bi-valva de carbón pirrolítico, con la cual se han obtenido muy buenos resultados, pero que no está libre de complicaciones ${ }^{2,17,30}$. Nuestra experiencia muestra que las complicaciones derivadas de ésta prótesis fueron responsables de $19 \%$ de la mortalidad alejada de los pacientes. No obstante, la supervivencia alejada de los tres grupos fue muy satisfactoria y coincide con lo encontrado por otros autores ${ }^{17,30,37}$.

Concluimos que los factores de riesgo significativos en el resultado del reemplazo valvular mitral son dependientes de las características clínicas del paciente, no del tipo de intervención ni del sistema de salud de origen. La mortalidad 
operatoria fue menor que en estudios previos y refleja una mejor calidad del servicio prestado. Estimamos que la integración de los sistemas de salud público, universitario y privado es una alternativa eficiente para resolver adecuadamente patologías complejas.

\section{Referencias}

1. Rahimtoola SH. Lessons learned about the determinants of the results of valve surgery. Circulation 1988; 78: 1503-7.

2. Grunkemeier GL, Li HH, Naftel DC, Starr A, Rahimtoola SH. Long-term performance of heart valve prostheses. Curr Probl Cardiol 2000; 25: 73-154.

3. Rahimtoola SH. Choice of prosthetic heart valve for adult patients. J Am Coll Cardiol 2003; 41: 893-904.

4. Ray AA, Buth KJ, Sullivan JA, Johnston DE, Hirsch GM. Waiting for cardiac surgery: Results of a risk-stratified queuing process. Circulation 2001; 104: 192-8.

5. Plomp J, Redekop WK, Dekker FW, van Geldorp TR, Haalebos MM, Jambroes G, et al. Death on the waiting list for cardiac surgery in the Netherlands in 1994 and 1995. Heart 1999; 81: 593-7.

6. Morán S. Reflexiones sobre la carencia de especialistas. Rev Chil Cir 2012; 64: 333-4.

7. Greenberger NJ, Davies NE, Maynard EP, Wallerstein RO, Hildreth EA, Clever LH. Universal access to health care in America: A moral and medical imperative. Ann Intern Med 1990; 112: 637-9.

8. American College of Physicians. Access to health care. Position paper. Ann Intern Med 1990; 112: 641-61.

9. Morán S. Tratamiento quirúrgico de las valvulopatías adquiridas. En: Oyonarte M, Román O, Corbalán R, eds. Enfermedades del corazón y de los vasos. Santiago: $4^{\circ}$ Edición. Editorial Mediterráneo Ltda; 2013. p. 479-90.

10. Grover FL, Hammermeister KE, Burchfiel C. Initial report of the veterans' administration preoperative risk assessment study for cardiac surgery. Ann Thorac Surg 1990; 50: 12-28.

11. Akins CW, Miller DC, Turina MI, Kouchoukos NT, Blackstone EH, Grunkemeier GL, et al. Guidelines for reporting mortality and morbidity after cardiac valve interventions. J Thorac Cardiovasc Surg 2008; 135: 732-8.

12. The Criteria Committee of the New York Heart Association: Nomenclature and criteria for diagnosis. $9^{\text {th }} \mathrm{ed}$. Boston, Little, Brown, 1994.

13. American College of Cardiology. Left ventricular ejection fraction assessment. 2012. http://www.cardiosource. org.

14. Enríquez-Sarano M, Tajik AJ, Schaff HV, Orszulak TA,
Bailey KR and Frye RL. Echocardiographic prediction of survival after correction of organic mitral regurgitation. Circulation 1994; 90: 830-7.

15. Hellgren L, Kvidal P, Horte LG, Krusemo UB, Stahle E. Survival after mitral valve replacement: Rationale for surgery before occurrence of severe symptoms. Ann Thorac Surg 2004; 78: 1241-7.

16. Teoh KH, Ivanov J, Weisel RD. Determinants of survival and valve failure after mitral valve replacement. Ann Thorac Surg 1990; 49: 643-8.

17. Emery RW, Krogh CC, Arom KV, Emery AM, BenyoAlbrecht K, Joyce LD, et al. The St. Jude medical cardiac valve prosthesis: A 25-year experience with single valve replacement. Ann Thorac Surg 2005; 79: 776-82.

18. Thourani VH, Weintraub WS, Craver JM, Jones EL, Gott JP, Brown WM, et al. Influence of concomitant CABG and urgent/emergent status on mitral valve replacement surgery. Ann Thorac Surg 2000; 70: 778-84.

19. Clínicas Privadas AG. Dimensionamiento de la atención de salud en Chile. 2012.

20. Carroll RJ, Horn SD, Soderfeldt B, James BC, Malmberg L. International comparison of waiting times for selected cardiovascular procedures. J Am Coll Cardiol 1995; 25: 557-63.

21. Ministerio de Salud. Objetivos sanitarios para la década 2000-2010. Una evaluación a la mitad del período. http://epi.minsal.cl/epi/html/sdesalud/OS/EvOS_III.pdf.

22. Rexius H, Brandrup-Wognsen G, Oden A, Jeppsson A. Mortality on the waiting list for coronary artery bypass grafting: Incidence and risk factors. Ann Thorac Surg 2004; 77: 769-74.

23. MINSAL. Encuesta nacional de salud 2009-2010. http:// wwwminsalgobcl/portal/url/item/bcb03d7bc28b64dfe040010165012d23pdf. 2011.

24. Dubernet J, Chamorro G, Maturana G, Morán S, Urzúa J, Durán F, et al. Seguimiento a largo plazo de pacientes con reemplazo aislado de válvula mitral. Rev Med Chile 1979; 107: 603-9.

25. Braun S, Chamorro G, Dubernet J, Irarrázaval MJ, Maturana G, Morán S, et al. Evolución alejada del reemplazo mitral con la válvula de Bjork-Shiley. Rev Med Chile 1987; 115: 214-9.

26. Scott WC, Miller DC, Haverich A, Mitchell RS, Oyer $\mathrm{PE}$, Stinson EB, et al. Operative risk of mitral valve replacement: Discriminant analysis of 1329 procedures. Circulation 1985; 72 (suppl II): II-108-II-119.

27. Khan SS, Trento A, De Robertis M, Kass RM, Sandhu $\mathrm{M}$, Czer LS, et al. Twenty-year comparison of tissue and mechanical valve replacement. J Thorac Cardiovasc Surg 2001; 122: 257-69.

28. Jamieson WR, Edwards FH, Schwartz M, Bero JW, Clark 
RE, Grover FL. Risk stratification for cardiac valve replacement. National cardiac surgery database. Database committee of the Society of Thoracic Surgeons. Ann Thorac Surg 1999; 67: 943-51.

29. Thourani VH, Weintraub WS, Craver JM, Jones EL, Mahoney EM, Guyton RA. Ten-year trends in heart valve replacement operations. Ann Thorac Surg 2000; 70: 44855.

30. Remadi JP, Bizouarn P, Baron O, Al Habash O, Despins P, Michaud JL, et al. Mitral valve replacement with the St. Jude medical prosthesis: A 15-year follow-up. Ann Thorac Surg 1998; 66: 762-7.

31. Chan V, Burwash IG, Lam B-K, Auyeung BS, Tran A, Mesana TG, et al. Clinical and echocardiographic impact of functional tricuspid regurgitation repair at the time of mitral valve replacement. Ann Thorac Surg 2009; 88: 1209-15.

32. Morán S. Consideraciones acerca del reemplazo valvular aórtico. Rev Chil Cardiol 2007; 26: 448-50.

33. Bonow RO, Carabello BA, Chatterjee K, de León AC, Jr., Faxon DP, Freed MD, et al. 2008 Focused update incorporated into the ACC/AHA 2006 guidelines for the management of patients with valvular heart disease: A report of the American College of Cardiology/American Heart Association task force on practice guidelines. J Am Coll Cardiol 2008; 52: e1-e142.

34. Gammie JS, Sheng S, Griffith BP, Peterson ED, Rankin JS, O’Brien SM, et al. Trends in mitral valve surgery in the United States: Results from the Society of Thoracic Surgeons adult cardiac surgery database. Ann Thorac Surg 2009; 87: 1431-7.

35. Murday AJ, Hochstitzky A, Mansfield J, Miles J, Taylor B, Whitley E, et al. A prospective controlled trial of St. Jude versus Starr-Edwards aortic and mitral valve prostheses. Ann Thorac Surg 2003; 76: 66-73.

36. Godje OL, Fischlein T, Adelhard K, Nollert G, Klinner W, Reichart B. Thirty-year results of Starr-Edwards prostheses in the aortic and mitral position. Ann Thorac Surg 1997; 63: 613-9.

37. Bando K, Kobayashi J, Hirata M, Satoh T, Niwaya K, Tagusari $\mathrm{O}$, et al. Early and late stroke after mitral valve replacement with a mechanical prosthesis: Risk factor analysis of a 24-year experience. J Thorac Cardiovasc Surg 2003; 126: 358-64. 